\title{
Symmetrical Transition Waveform Control on Double-Wire MIG Welding
}

Article in Journal of Materials Processing Technology · September 2015

DOI: 10.1016/j.jmatprotec.2015.08.031

CITATIONS

2

5 authors, including:

\section{Kang Zhou}

Chinese Academy of Sciences

18 PUBLICATIONS 77 CITATIONS

SEE PROFILE

Some of the authors of this publication are also working on these related projects:

resistance spot welding research View project 


\title{
Symmetrical transition waveform control on double-wire MIG welding
}

\author{
Yao Ping $^{\mathrm{a}}$, Xue Jiaxiang ${ }^{\mathrm{b}}$, Zhou Kang ${ }^{\mathrm{c}, *}$, Wang Xiaojun ${ }^{\mathrm{a}}$, Zhu Qiang ${ }^{\mathrm{b}}$ \\ a College of Electromechanical Engineering, Guangdong Polytechnic Normal University, Guangzhou 510635, China \\ ${ }^{\mathrm{b}}$ School of Mechanical and Automotive Engineering, South China University of Technology, Guangzhou 510640, China \\ ' State Key Laboratory of High-temperature Gas Dynamics, Institute of Mechanics, Chinese Academy of Sciences, Beijing 100190, China
}

\section{A R T I C L E I N F O}

\section{Article history:}

Received 6 May 2015

Received in revised form 27 August 2015

Accepted 29 August 2015

Available online 11 September 2015

\section{Keywords:}

Double-wire MIG welding

Symmetrical transition

Current waveform control

Orthogonal experiment

\begin{abstract}
A B S T R A C T
To reduce unsteady and undercut phenomena during double-wire MIG welding process, a symmetrical transition waveform control method, which was based on analyzing the effect of the electromagnetic force, was proposed. A symmetrical transition period was added when the welding current was switched between peak value and base value. Three variables were introduced, which were leading transition current $I_{\mathrm{SL}}$, double wires transition time $T_{\mathrm{S}}$ and trailing transition current $I_{\mathrm{ST}}$. Corresponding experiments were conducted to compare the different phenomena between employing this period and not employing the period. Orthogonal experiments were conducted to study the influence by the three variables. An optimum combination of $I_{\mathrm{SL}}, T_{\mathrm{S}}, I_{\mathrm{ST}}$ could be obtained by means of range analysis, and corresponding experiments verified the combination. All the experiments showed that the symmetrical transition period can reduce the large variation of welding current, make the welding process steadier and the welding seam much more polished, and obtain welding seam with higher quality.
\end{abstract}

(C) 2015 Elsevier B.V. All rights reserved.

\section{Introduction}

Double-wire MIG welding is an efficient welding method that has been increasingly employed in the welding industry. In the 1950s, Ashton (1954) and Steinert (1954), separately proposed the systematic structure of double-wire MIG welding, which laid foundations for current double-wire MIG power source application. Person and Ruzek (1956) improved the double-wire MIG welding system, and proposed a tandem system of arc welding. Because two wires simultaneously provide heat energy to the base metal, compared to single-wire welding technology, tandem welding can change the heat distribution, and effectively avoid undercut and has various other relative disadvantages. Hence, tandem welding can improve the travel speed for automatic welding and yield welding seams with high quality (Li and Zhang, 2010).

As a highly productive welding technique, double-wire MIG welding appeals a lot of focuses. Reis et al. (2015) focused on the effect of different elements on arc interruption in double-wire MIG welding, and concluded that the proper selections of certain parameters, such as a little delay between trailing current and leading

\footnotetext{
* Corresponding author. +861082545985.

E-mail addresses: ypsunny@163.com (P. Yao), zhoukang326@126.com, zhoukang@imech.ac.cn (K. Zhou).
}

current, can lower the possibility of the appearance of arc interruption. Niu et al. (2010) focused on the effect of the peak pulse voltage of the leading current on the welding process and weld quality. Shi et al. (2014a,b) established a model to simulate the double-wire MIG welding process, and respectively obtained the effects of arc length and welding current on the process. Wen et al. $\underline{(2010)}$ analyzed the different effects of synchronous versus alternative changes in host and accessory pulse currents. Because the double-wire MIG welding process involves many different control variables and complex techniques, current researches have mainly focused on a case in which the leading current and trailing current have the same frequency but inverse phase (SFIP). Many previous studies have concluded that under the SFIP current, the noise of the output electrical arc is low and the welding process is steady; however, the welding penetration is relatively small(Andersson et al., 2006; Bagus Yudharto Bharotokusumo et al., 2008). Conversely, Ueyama et al. (2005) conducted an experiment with the leading current and trailing current having the same frequency and same phase (SFSP), and then they found that when the peak period of the trailing current was delayed by $0.5 \mathrm{~ms}$ more than that of the leading current, a steady welding process together with a satisfactory welding seam could be obtained. Moreover, by delaying the pulse end timing of the trailing arc by $0.4-0.5 \mathrm{~ms}$ from that of the leading arc, Ueyama et al. (2009) work's effectively reduced the arc interference in tandem pulsed gas metal arc welding. 
Additionally, Motta et al. (2007) showed that an inverse phase waveform is not a necessary condition for obtaining satisfactory welding seams. Wang et al. (2011) discovered that deceasing the ratio between the leading current and the trailing current could reduce the possibility of arc interruption.

Previous studies have examined different aspects of doublewire MIG welding using various methods, and have drawn some interesting conclusions. However, the current waveform of doublewire MIG welding that is typically employed is a traditional, rectangular type. Because the difference between the base value and the peak value of the rectangular signal is very large, unsteady welding process may be induced, which can make the variation in energy during the different welding stages difficult to properly control. As the processor capacity and welding machine digital technologies have been improved in past decade, the new current waveform control method was more and more considered, so that the molten droplet transferring can be well controlled, as well as the welding products with high quality can be correspondingly obtained. Lincoln Electric Co. developed a surface tension transfer (STT) process, which accurately controlled the current in seven points of each waveform cycle period. This method substantially reduced spatter and lowered heat delivery on thin-gauge material (DeRuntz, 2003). Miller Electric Mfg. Co. introduced a new welding technology process called Regulated metal deposition (RMD) process. The technology was based on an advanced software application for modified short circuit transfer GMAW (MIG welding) that monitored the electrode current in each step of the short circuiting. The RMD approach was illustrated in different steps as follows: Wet, Pinch, Clear, Blink, Ball, Background, Pre-short, which can predict future arc conditions and controls the droplet transfer accordingly (Peterson, 2009). Apart from welding line pipe carbon steel materials, the technology can also be used in welding nickel alloy (Petro, 2011). Kemppi Company developed a modified short arc circuit welding process, which was called WiseRoot Process. In this process, the power source was monitored by the wave of the current, which can be analyzed in two main parts: the short circuit and the arc period (Uusitalo, 2007). This process was capable of achieving similar welds with 5-25\% less heat delivery than the conventional short arc and can maintain the same heat delivery as a laser welding process (Peltola et al., 2010). Fronius Company combined special wave control features and an assistance backdrawing force, and then developed a cold metal transfer (CMT) process which can be used in aluminium alloy materials welding (Schierl, 2005). Also, Feng et al. (2009) employed the sensing and image method to analyze the wave control characteristics and its droplet transfer process in CMT method, the results showed that it can realize no-spatter welding and low heat input during welding process. Daihen Corporation (Tong and Ueyama, 2011) introduced a method of low frequency modulated type pulsed MIG welding process, which can improve the welding quality by switching the high frequency and low frequency pulse in real time. Yao et al. (2009) explored the application of this control method on aluminium alloy materials welding, and found that strong/weak pulse peak current and high-frequency have great influence on the stability of welding process and spatters. Peters (2013) introduced a power supply which includes a waveform type selector that selects a desired shape for an output waveform, the operator is given a choice between an advanced "crisp" square wave, a "soft" square wave, a sine wave, and a triangle wave, which can meet the requirements in different welding occasions. Kawagoe and Suzuki (2014) developed a power source which can control the welding current in real time. A current increasing phase and a current decreasing phase were added in the latter of pulse peak phase in this process. The current increasing can melt the workpiece. Hence, this method can obtain a satisfactory welding performance by low energy delivery.
Though there were many current waveform methods proposed to improve the welding technique, they mainly focused on the single-wire MIG application (Kah et al., 2013). It is because that the current double-wire MIG facility usually employed two separated control parts, and then the communication between two parts is necessary to conduct online corporative control. However, real time control must be guaranteed in welding process control, which needs high-speed Central Processing Unit (CPU). Also, achieving waveform coordinated control for the two parts were very complex. Hence, there were less relative research about double-wire MIG current waveform control process in reality. Currently, the processing capacity of CPU has been largely increased, whose capacity can be capable of processing control actions in two circuits. Under this circumstance, the current waveform control process is proposed in this paper so as to improve the performance of double-wire MIG technique and the steady of the process.

In this work, according to the current double-wire tandem welding technology, a different current waveform control process, which was symmetrical transition waveform control method, was proposed to improve the double-wire MIG welding quality and operation. A significant characteristic of this method was that a symmetrical transition period was newly introduced, which induced that the magnitude of energy variation was reduced during the switching process between peak value and base value of the current. After fine tuning the relative parameters in this period, much steadier welding process can be obtained. This method was further studied step by step by means of a series of experiments, such as experiments which employed or not employed the symmetrical transition period, orthogonal experiments to seek the affecting discipline of parameters in the transition period, as well as verification experiment of optimum parameters combinations obtained by range analysis. Final results showed that a much steadier welding process and much more polished welding seam can be obtained using this proposed method.

\section{Principle of double-wire MIG welding}

The double-wire MIG welding technique involves placing two wires on a specially designed welding torch in a predetermined pattern; the two wires are fed by individual power sources and insulated from each other. Almost all of parameters of the two wires including the wire feed speeds are independent from each other in reality. Both of the diameters and materials of the wires may be different. For example, one wire can be fed by an impulse signal, and a different signal type can feed the other wire. During the process, the electrical arc can be generated in the terminates of the wires, and then the heat generated by electrical arc discharging can melt the wire and base metal together, finally, a weld seam will formed after the heat removed. Hence, to obtain satisfactory performance, the electrical arc should be well-controlled using this design and some satisfactory performances, such that the mutual interferences of the two wires should be low (Goecke et al., 2011), can be correspondingly obtained. Fig. 1 shows a schematic diagram of double-wire MIG welding.

In double-wire MIG welding, the distance between the two direct current (DC) electrical arcs is very small. Under this circumstance, the magnetic interference between the electrical arcs may induce an unsteady welding process. Apart from the concern regarding the metal transfer process of each wire, the mutual effects from each wire should also be seriously considered. Hence, controlling of the current waveform of each wire is very important for guaranteeing the efficiency and steadiness of the double-wire MIG welding process.

In this system, the two wires are independent from each other and metal transfer occurs in the same molten pool. However, 


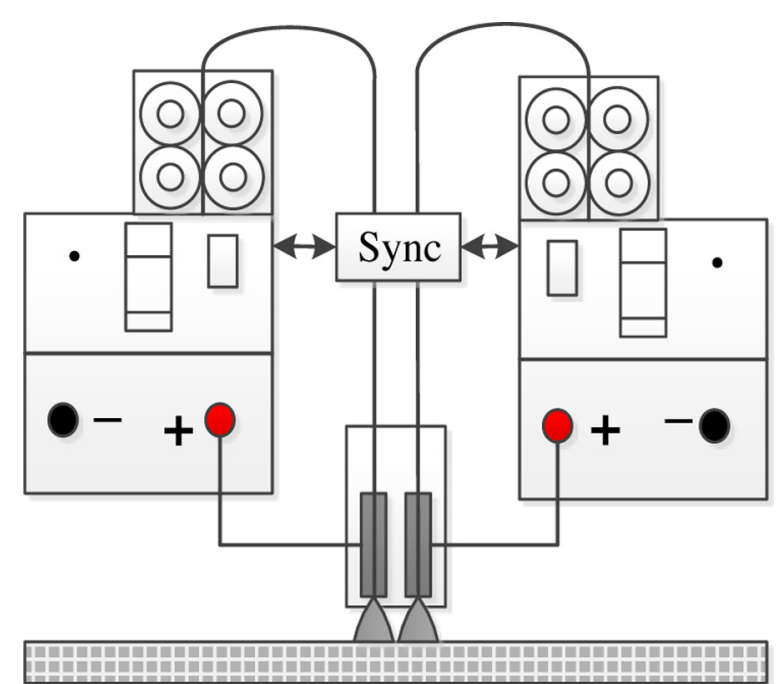

Fig. 1. Schematic figure of double-wire MIG welding.

because the arc is both a heat source and a force source, the mutual interference between the two wires may affect the welding process, which induce more metal transfer characteristics than that in single-wire MIG welding.

To address this issue, Halmoy (1980), Chandel (1987, 1988) and Suban and Tušek (2001) studied the melting speed and believed that melting was induced by both arc heat and resistance heat. Tušek (2000) established an equation for calculating the melting rate:

$M=a_{0}+a_{1} I+a_{2} \frac{I^{2} L}{d^{2}}$

where $a_{0}, a_{1}$, and $a_{2}$ are constants. The units of the parameters in Eq. (1) can be expressed in the following units: $M(\mathrm{~kg} / \mathrm{h}), I(A$ per wire), $L(\mathrm{~mm})$, and $d(\mathrm{~mm})$. It can be observed that the melting rate $M$ is determined by the welding current intensity $I$, wire extension length $L$ and wire diameter $d$. When other conditions remain unchanged, a high welding current indicates a high melting rate and a high welding efficiency.

The metal transfer process is affected by surface tension, the electromagnetic shrinkage force, the plasma fluid force and gravity. Because the distance between the two wires in double-wire MIG welding is very small, a mutually attracting electromagnetic force can be induced between the two wires, which may induce the magnetic arc blow phenomenon. This phenomenon causes the arcs to present an oblique, conical shape or bundle, and the arc stiffness becomes poor.

The electromagnetic force may affect the electromagnetic shrinkage force and the plasma fluid force, which may change the arc during welding. If the change is significantly large, arc interruptions, irregular metal transfer or considerable expulsion may be induced. The magnitude of the electromagnetic force is related to the current of the wire, as well as the angle and the distance between the two wires. Ueyama et al. (2007) established an equation for calculating the electromagnetic force between two wires.

$F=F_{\mathrm{ML}}=F_{\mathrm{MT}}=\left(\frac{I_{\mathrm{T}}}{\pi r_{\mathrm{T}}^{2}}\right) \times\left(\frac{\mu_{0} I_{\mathrm{L}}}{2 \pi D_{\mathrm{E}}}\right)$

where $F_{\mathrm{MT}}$ and $F_{\mathrm{ML}}$ are the electromagnetic force (Lorentz force), $I_{\mathrm{L}}$ is the leading current, $I_{\mathrm{T}}$ is the trailing current, $\mu_{0}$ is the permeability of free space, $D_{\mathrm{E}}$ is the distance between the two wires, and $r_{\mathrm{T}}$ is the radius of the trailing arc column. Eq. (2) indicates that the product of the currents in the two wires determines the electromagnetic force when the other physical conditions remain unchanged.

Hence, to reduce the mutual interference of the two wires, the method of decreasing the magnitude of the welding current can be employed. However, decreasing the welding current can also reduce the amount of energy delivered, the melting rate, and the welding efficiency, which may cause the double-wire MIG welding to lose its merit as a highly efficient process. In this case, the method of waveform control is proposed to improve the stability of the double-wire MIG welding process.

\section{Proposed current waveform control method}

In general case, three control strategies are employed in doublewire MIG welding, as shown in Fig. 2(a)-(c).

The number of control variables is very large because of the complexity of the current waveform for double-wire pulse welding. The key control variables are shown in Fig. 2(a): the leading peak current $I_{\mathrm{Lp}}$, leading base current $I_{\mathrm{Lb}}$, leading peak time $T_{\mathrm{Lp}}$, leading base time $T_{\mathrm{Lb}}$, trailing peak current $I_{\mathrm{Tp}}$, trailing base current $I_{\mathrm{Tb}}$, trailing peak time $T_{\mathrm{Tp}}$, trailing base time $T_{\mathrm{Tb}}$, leading current frequency $f_{\mathrm{L}}$ and trailing current frequency $f_{\mathrm{T}}$. All variables are independently adjusted and controlled.

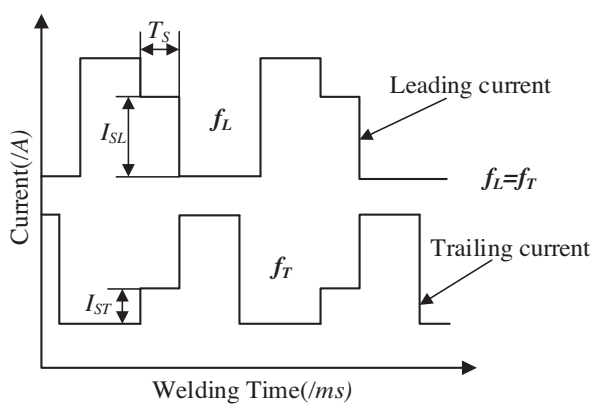

Fig. 3. Symmetrical transition double-wire waveform.
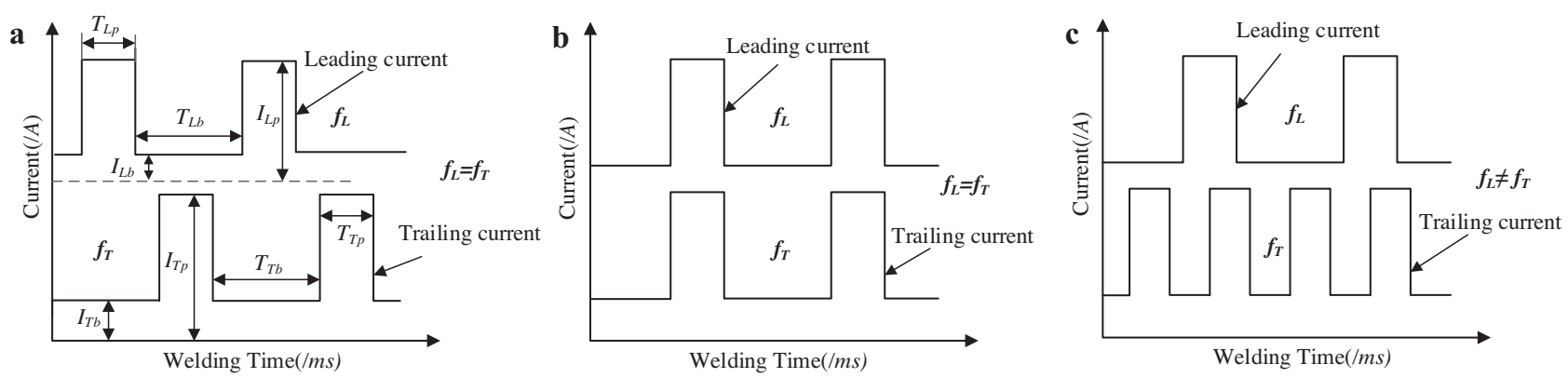

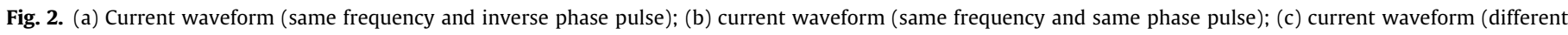
frequency and different phase pulse). 


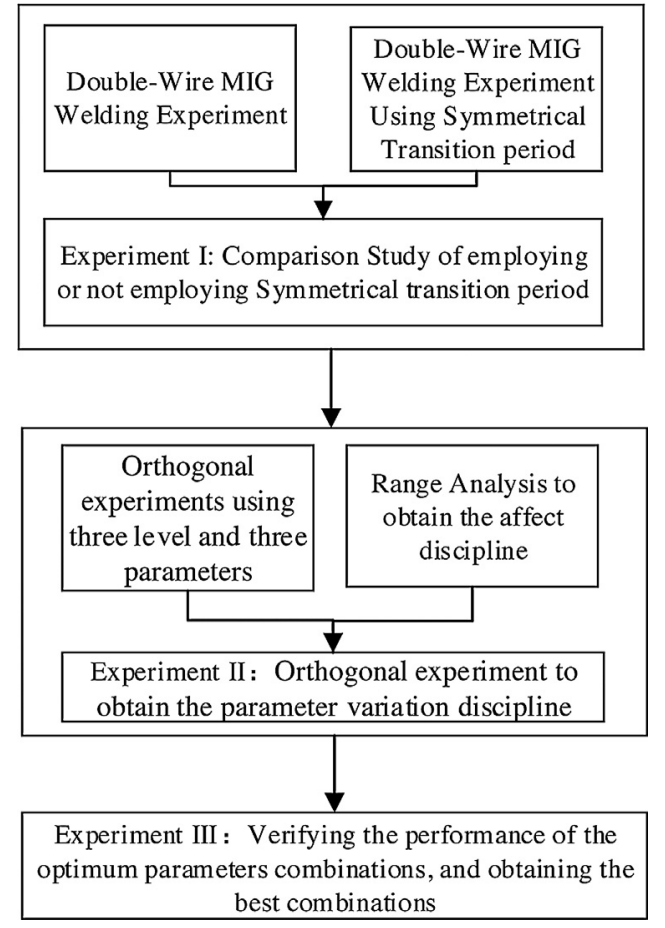

Fig. 4. Flowchart of the experimental procedures.

Fig. 2(b) shows the current waveform under the same frequency and same phase. Under this circumstance, the electromagnetic force is large because the phases of the two wires are identical, which induces significant mutual interference:

$\beta=\left(\frac{1}{\pi r_{\mathrm{T}}^{2}}\right) \times\left(\frac{\mu_{0}}{2 \pi D_{\mathrm{E}}}\right)$

$F_{1}=\beta \times\left(a_{\mathrm{L}} I_{\mathrm{Lav}} \times a_{\mathrm{T}} I_{\mathrm{Tav}}\right)\left(a_{\mathrm{T}}, a_{\mathrm{L}}>1\right)$

$F_{1}=\beta a_{\mathrm{L}} a_{\mathrm{T}} \times\left(I_{\mathrm{Lav}} \times I_{\mathrm{Tav}}\right)$

$I_{\mathrm{Lp}}=a_{\mathrm{L}} I_{\mathrm{Lav}}$

$I_{\mathrm{Tp}}=a_{\mathrm{T}} I_{\mathrm{Tav}}$

where $I_{\text {Lav }}$ is the mean value of leading current, $I_{\text {Tav }}$ is the mean value of trailing current; $a_{\mathrm{L}}$ and $a_{\mathrm{T}}$ are the coefficients of the peaks of the leading current and trailing current, respectively.

As shown in Fig. 2(c), when the current waveform is under a random mode involving different frequencies and different phases, the mutual interference may also be very large because the phases of the two wires may be identical during the welding process. Moreover, the welding process is difficult to control because the phases of the two wires are random, which provides no rules for metal transfer. Under these conditions, the largest electromagnetic force is identical to that in Fig. 2(b).

On the other hand, as shown in Fig. 2(a), when the current waveform exhibits the same frequency with a $180^{\circ}$ phase difference, meaning that there are regular and alternative phases of the two arc effects, the effect of the force on the metal transfer is very low and the mutual inference can be solved for. However, reducing the current from the large peak value to the small base value may easily induce an unsteady welding process or arc interruption. Hence, it can be concluded that during the double-wire MIG welding process, difference of phases and values between the current peak and current base can directly affect the steadiness of the welding process.

Under the same melting rate, decreasing the mutual force between two wires benefits the arc stability, and reduces the amount of heat delivered. Hence, the mutual interference between two wires can be reduced by the pulse welding method:

$F_{2}=\beta a_{\mathrm{L}} b_{\mathrm{T}} \times\left(I_{\mathrm{Lav}} \times I_{\mathrm{Tav}}\right) \quad\left(b_{\mathrm{T}}<1\right)$

where $b_{T}$ is the scaling factor between the base value and the average value of the trailing current. Clearly, when the phases of two wires are inverted, the electromagnetic force $F_{2}$ is lower than that observed when the phases are the same, $F_{1}$ in Eqs. (4), (5) and (8).According to this analysis, when the current values are not very large, a satisfactory welding seam can be obtained under the waveform with the same frequency and the same phase, and the possibility of the appearance of arc interruption can also be simultaneously reduced. However, because the current value is insufficient for supplying enough energy, the welding efficiency is low. To reduce the effect of the electromagnetic force and improve the welding efficiency, the symmetrical transition waveform control method is proposed as shown in Fig. 3.

It can be shown in Fig. 3 that in the proposed method, a transition period is introduced when the current phase is changed. Hence, the entire pulse waveform can be described by three components: peak period, transition period and based period.

Correspondingly, three variables are added: the leading transition current $I_{\mathrm{SL}}$, double wires transition time $T_{\mathrm{S}}$ and trailing transition current $I_{\mathrm{ST}}$. These variables can directly determine the effects of the energy delivery and electromagnetic force on metal transfer during the transition period.

The electromagnetic force in the symmetrical transition period can be calculated as follows:

$F_{\mathrm{S}}=\beta \mathrm{s}_{\mathrm{L}} \mathrm{S}_{\mathrm{T}} \times\left(I_{\mathrm{Lav}} \times I_{\mathrm{Tav}}\right)$

$I_{\mathrm{SL}}=S_{\mathrm{L}} \times I_{\mathrm{Lav}}$

$I_{S T}=\mathrm{S}_{\mathrm{T}} \times I_{\mathrm{Tav}}$

where $s_{\mathrm{L}}$ is the leading current transition coefficient, and $s_{\mathrm{T}}$ is the trailing current transition coefficient. $s_{\mathrm{T}}, s_{\mathrm{L}}$ may be greater or smaller than 1 . When $s_{\mathrm{L}}=s_{\mathrm{T}}=1, I_{\mathrm{SL}}=I_{\mathrm{Lav}}, I_{\mathrm{ST}}=I_{\mathrm{Tav}}$, and the symmetrical transition period is added, according to Eq. (1), I remains unchanged, and then the mean value of the current and melting rate remain unchanged; however, because $a_{\mathrm{L}} \times b_{\mathrm{T}}$ may be greater than $s_{\mathrm{L}} \times s_{\mathrm{T}}$, the value of electromagnetic force in the symmetrical transition period, which is marked as $F_{\mathrm{s}}$, may be lower than that observed when the phases of the two arcs are inverted.

By adjusting these variables and the relationship between $s_{\mathrm{L}}$ and $s_{\mathrm{T}}$, the electromagnetic force under the same phase mode of the two wires can approach or even be less than that under the inverse phase mode. Hence, according to above analysis, the existence of a symmetrical transition period can make the welding process much steadier and controllable, and then a more satisfactory welding quality can be obtained (Yao et al., 2012).

\section{Experimental setup and method}

\subsection{Experimental setup}

To verify the proposed method, actual welding experiments were conducted. In the experiment, a DSP integrated double-wire MIG power source, a walking controller, a welding experiment platform, a welding arc dynamic wavelet analysis instrument, a double-wire pulse MIG welding inverter with a software switch, a wire feeder, a double-wire welding torch, a tank and other relative facilities were employed. During the experimental process, the welding arc dynamic wavelet analysis instrument was employed to collect and analyze the waveform, while the integrated double- 
Table 1

Control parameters used in the current waveform.

\begin{tabular}{llllllll}
\hline Parameter & 340 & 88 & 300 & 75 & 5.2 & 12.5 & 56 \\
Item & $I_{\mathrm{Lp}}(\mathrm{A})$ & $I_{\mathrm{Lb}}(\mathrm{A})$ & $I_{\mathrm{Tp}}(\mathrm{A})$ & $I_{\mathrm{Tb}}(\mathrm{A})$ & $T_{\mathrm{p}}(\mathrm{ms})$ & $T_{\mathrm{b}}(\mathrm{ms})$ & $f_{\mathrm{L}}, f_{\mathrm{T}}(\mathrm{Hz})$ \\
\hline
\end{tabular}

wire pulse MIG welding inverter was used to control the actual waveform.

The basic conditions used in the double-wire symmetrical transition current waveform control experiment were as follows: the workpiece was Q235 steel with a thickness of $8.0 \mathrm{~mm}$. The welding wires were composed of H08Mn2SiA and measured $1.0 \mathrm{~mm}$ in diameter, and the shielding gas was pure $\operatorname{argon}(15 \mathrm{~L} / \mathrm{min}$ flow). The distance between the two terminals of the wires was $8.0 \mathrm{~mm}$. In addition, flat welding was used. The control parameters used in the current waveform are shown in Table 1.

\subsection{Experimental program}

To comprehensively explore the operational performance of symmetrical transition waveform control method, three experiments were employed as follows:

1) The first experiment was to compare the different phenomena that occurred when the symmetrical transition period was employed and not employed, the goal of the experiment was to observe the effect of the symmetrical transition period on the process and performance.

2) The second experiment was the orthogonal experiment employing three parameters with three different values under three different levels. Then the range analysis was employed to further study the effect of the parameters in the symmetrical transition period on the welding quality.

3) Different parameters combinations were employed in the experiments so as to verify the effectiveness of the range analysis and obtain an optimum parameters combination.

To clearly show the procedures, Fig. 4presents a flowchart of the above experiments.

\section{Experiments and analysis}

\subsection{Experiment of symmetrical transition period}

First, two experiments, one employed the transition period and the other did not employ the period, were conducted and compared to explore the effect of symmetrical transition period on the welding process. The data used in these experiments are shown in Table 2.

The average value of welding current could be obtained as follows: when the transition period was not employed, the average

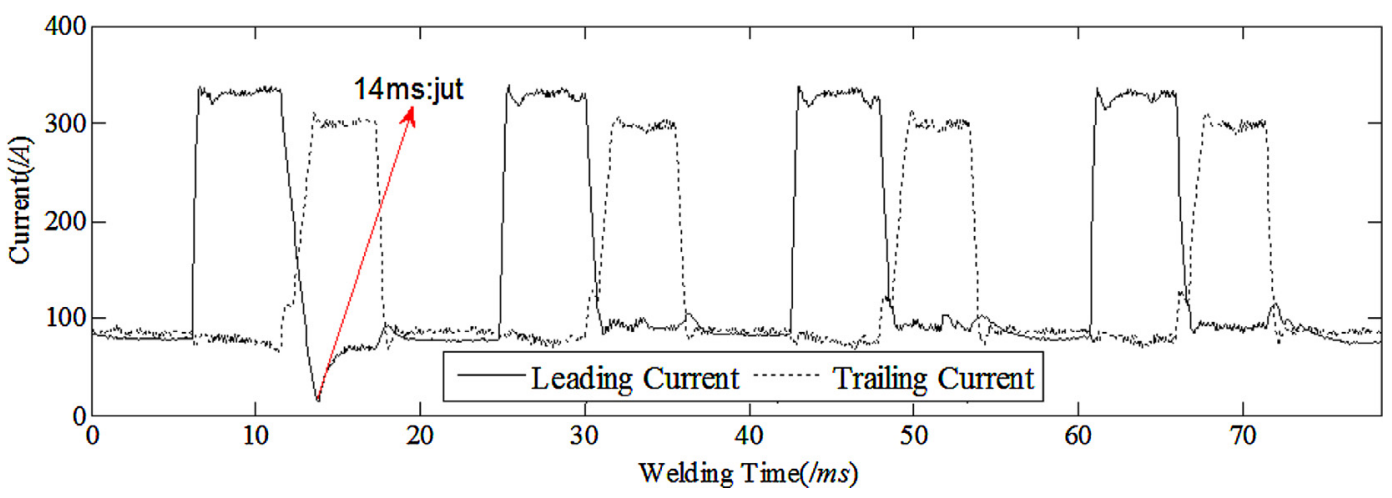

Fig. 5. The current waveform when the transition phase is not employed.

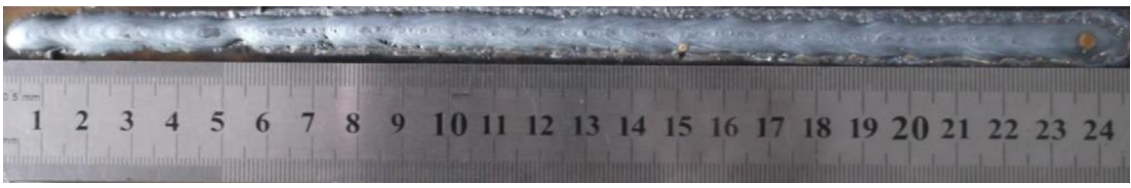

Fig. 6. Welding seam when the transition phase is not employed.

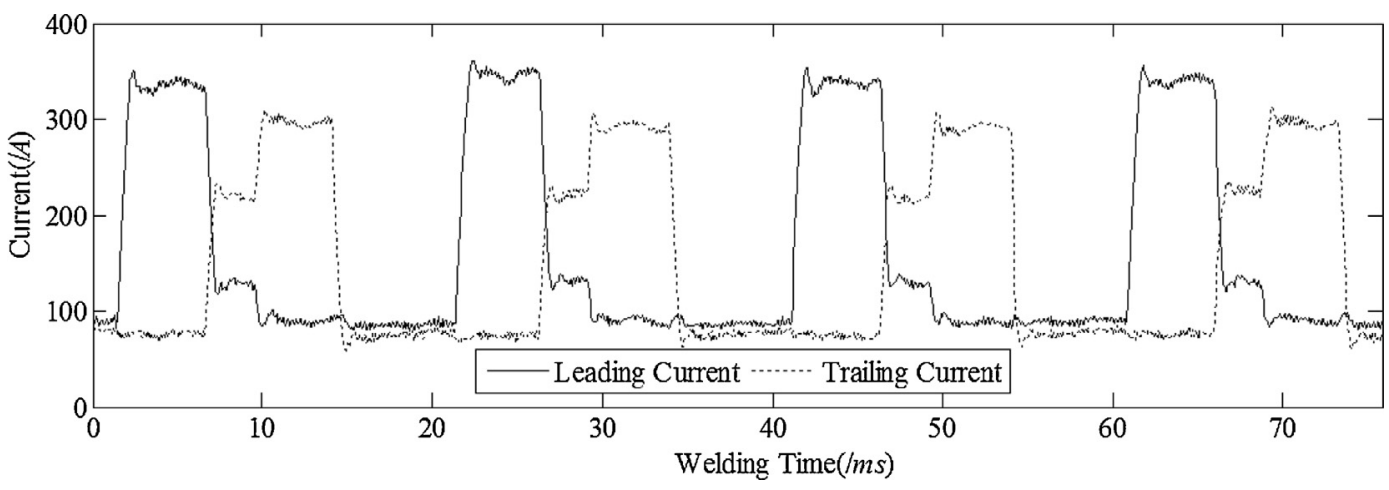

Fig. 7. The current waveform and seam when the transition phase is employed. 
Table 2

Experimental data of using/not using symmetrical transition period experiment.

\begin{tabular}{|c|c|c|c|c|}
\hline Item & Peak time $T_{\mathrm{p}}(\mathrm{ms})$ & Base time $T_{\mathrm{b}}(\mathrm{ms})$ & Transition parameters & Walking velocity $(\mathrm{m} / \mathrm{min})$ \\
\hline No using transition period & 5.2 & 12.5 & NA & 0.9 \\
\hline Using transition period & 5.2 & 12.5 & $\begin{array}{l}T_{\mathrm{S}}=3.2 \mathrm{~ms} \\
I_{\mathrm{SL}}=130 \mathrm{~A} \\
I_{\mathrm{ST}}=220 \mathrm{~A}\end{array}$ & 1.0 \\
\hline
\end{tabular}

values of leading current $I_{\text {Lav }}$ and the trailing current $I_{\text {Tav }}$ could be obtained using Eqs. (12) and (13), respectively:

$I_{\mathrm{Lav}}=\frac{I_{\mathrm{Lp}} \times T_{\mathrm{Lp}}+I_{\mathrm{Lb}} \times T_{\mathrm{Lb}}}{T_{\mathrm{Lp}}+T_{\mathrm{Lb}}}$

$I_{\mathrm{Tav}}=\frac{I_{\mathrm{Tp}} \times T_{\mathrm{Tp}}+I_{\mathrm{Tb}} \times T_{\mathrm{Tb}}}{T_{\mathrm{Tp}}+T_{\mathrm{Tb}}}$

when the transition period was employed, corresponding average values of two currents $I_{\text {SLav }}$ and $I_{\text {STav }}$ could be calculated by EqS. (14) and (15):

$I_{\text {SLav }}=\frac{I_{\mathrm{Lp}} \times T_{\mathrm{Lp}}+I_{\mathrm{Ls}} \times T_{\mathrm{Ls}}+I_{\mathrm{Lb}} \times T_{\mathrm{Lb}}}{T_{\mathrm{Lp}}+T_{\mathrm{Ls}}+T_{\mathrm{Lb}}}$

$I_{\mathrm{STav}}=\frac{I_{\mathrm{Tp}} \times T_{\mathrm{Tp}}+I_{\mathrm{Ts}} \times T_{\mathrm{Ts}}+I_{\mathrm{Tb}} \times T_{\mathrm{Tb}}}{T_{\mathrm{Tp}}+T_{\mathrm{Ts}}+T_{\mathrm{Tb}}}$

Then according to Table 1 and 2 together with Eqs. (12)-(15), $I_{\text {Lav }}$ and $I_{\text {Tav }}$ were $162.0 \mathrm{~A}$ and $141.1 \mathrm{~A}$, when the transition period was introduced, the corresponding currents values $I_{\text {SLav }}$ and $I_{\text {STav }}$ were $170.9 \mathrm{~A}$ and 139.4 A. By means of Eqs. (1) and (10)-(11), melting rate was increased, which induced the wire feed speed could be increased, therefore the welding efficiency was improved. The observed welding current waveform and welding seam when the transition phase was not employed are respectively shown in Figs. 5 and 6.

As shown in Fig. 5, when the transition phase was not employed and the current was directly switched between the base current and peak current, arc interruption could be easily induced because of the large variation in current. As shown in Fig. 5, at $14 \mathrm{~ms}$, the current waveform exhibited a distinct jut, which may have easily induced arc interruption. Additionally, the same feature could be observed in the welding seam. Due to the unsteady welding current, the shape of the welding seam was irregular, and some sunken areas appeared. After three sunken areas formed, the seam showed the formation of a grain, which may have been induced by restarting the arc, as well as the expulsion marks were clearly observed in the workpiece. Additionally, the arc length was irregular, and an exploding sound was heard during the process.

Figs. 7 and 8 show the current waveform and corresponding seam when the transition phase was employed.

It can be observed that the overall welding seam was regular. The arc length was consistent, and the length could be increased during the welding process. Moreover, no distinct arc interruption occurred, and the unsteady tendency could be observed. However, it could be immediately adjusted to the normal state. The sound heard during the experiment was different from that heard which occurred during the ordinary double-wire MIG welding process, which was "woon.". The sound head during the experiment was also much gentler, range between "woon." and "zi.". Furthermore, the shape of the seam was regular, and the surface gloss is much better than that observed when the transition phase was not employed. The crest of the seam was also different from that in an ordinary double-wire welding seam; the comparison is shown in Fig. 9. In an ordinary double-wire MIG welding seam, the crests appeared as fish-scale grains with only a few defects, as shown in the left panel of Fig. 9. Conversely, there was an obvious transition zone in the seam obtained by the symmetrical transition current waveform control method. This zone made the seam crest surface gloss much better than that of the ordinary seam. Additionally, the fish scale grain below the transition zone was much more regular and uniform, as shown in the right panel of Fig. 9.

According to the corresponding analysis, these results occurred during the transition period because a proper combination of leading and trailing transition currents filled the gap when the leading current and trailing current were switched. Hence, the flute between the fish-scale grains was filled with melting filler during the transition period, such that more better formed welding seam surface crest was obtained. It was because melting rate was increased by introducing the transition period, so that more liquid metal can fill the gap between fish-scale grains.

\subsection{Experiments of parameters affecting discipline}

\subsubsection{Orthogonal experiments}

Orthogonal experimental design is an important designing method to study the system effects that involves multiple factors and multiple levels. In this method, certain parameters are chosen as different factors for conducting experiments based on orthogonality. This selection can reduce the workload and involves corresponding methods of analyzing the experimental results, so as to yield more reliable conclusions (Keppel, 1991; Taguchi et al. 1987).

To further explore the effect of the transition period on doublewire MIG welding quality and obtain an optimum operational parameters combination, orthogonal experiments were conducted in this work.

In this experiment, the effects of the variation of three important parameters on welding quality were respectively explored; the parameters were leading transition current $I_{\mathrm{SL}}$, trailing transition current $I_{\mathrm{ST}}$ and current transition times $T_{\mathrm{S}}$. First, the variation ranges and levels of these three parameters could be confirmed using extreme experiments, and then an experimental scheme was designed using an orthogonal experiment table $L_{9}\left(3^{4}\right)$. Table 3 shows the parameters values employed corresponding three levels in the experiments.

Three repeated experiments were conducted. The waveform was collected and analyzed using the welding arc dynamic wavelet analysis instrument. The corresponding welding electrical signal was analyzed via the transient current waveform, the voltage-current probability density distribution and corresponding $U-I$ graph, the transient energy graph and other related methods. Additionally, the welding quality obtained from different combinations of key parameters could be assessed based on the observed phenomena and corresponding welding seam shapes. The welding quality could be scored using a previously reported quality scoring method (Yao et al., 2014a,b) and the data obtained from three repeated experiments using the same parameters. The detailed scheme and welding quality scores are shown in Table 4.

In the nine experiments described above, the mean current values of the two wires were: $I_{\text {SLav }}=154-180 \mathrm{~A}$ and $I_{\text {STav }}=131-151 \mathrm{~A}$. It was observed that welding could be steadily conducted with an unchanging walking velocity, with the other variables held at their standard values. No arc interruption or inability to initiate arc phenomena was observed; hence, the majority of the 


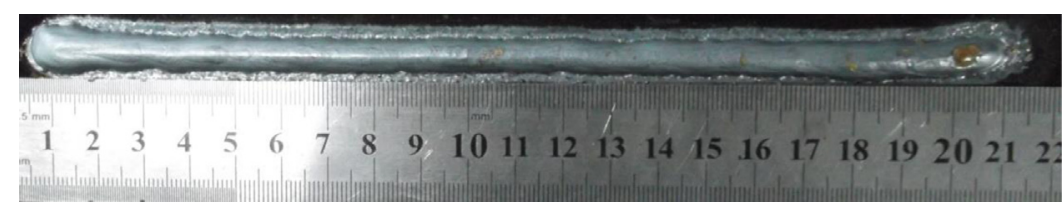

Fig. 8. Welding seam when the transition phase is employed.
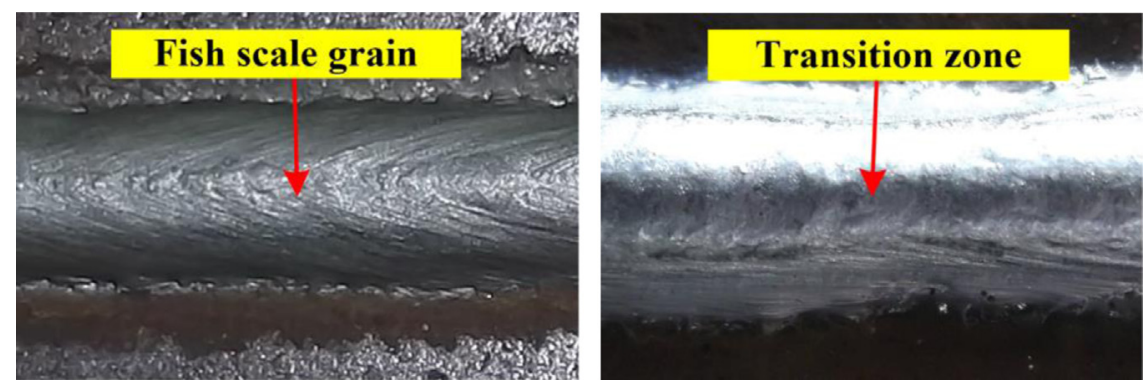

Fig. 9. Comparison between ordinary seam and seam obtained from symmetrical transition current waveform control method.

Table 3

Parameters and levels.

\begin{tabular}{|c|c|c|c|}
\hline Levels & Leading transition current $\left(I_{\mathrm{SL}} / \mathrm{A}\right)$ & Transition time $\left(T_{\mathrm{S}} / \mathrm{A}\right)$ & Trailing transition current $\left(I_{\mathrm{ST}} / \mathrm{A}\right)$ \\
\hline 1 & 130 & 0.9 & 120 \\
\hline 2 & 200 & 2 & 170 \\
\hline 3 & 250 & 3.2 & 220 \\
\hline
\end{tabular}

Table 4

Orthogonal test program and results.

\begin{tabular}{|c|c|c|c|c|}
\hline Index & $\begin{array}{l}\text { Parameters } \\
\text { Leading transition current }\left(I_{\mathrm{SL}} / \mathrm{A}\right)\end{array}$ & Transition time $\left(T_{\mathrm{S}} / \mathrm{A}\right)$ & Trailing transition current $\left(I_{\mathrm{ST}} / \mathrm{A}\right)$ & Welding quality \\
\hline 1 & 130 & 0.9 & 120 & 79 \\
\hline 2 & 130 & 2 & 170 & 68 \\
\hline 3 & 130 & 3.2 & 220 & 76 \\
\hline 4 & 200 & 0.9 & 170 & 74 \\
\hline 5 & 200 & 2 & 220 & 81 \\
\hline 6 & 200 & 3.2 & 120 & 76 \\
\hline 7 & 250 & 0.9 & 220 & 74 \\
\hline 8 & 250 & 2 & 120 & 85 \\
\hline 9 & 250 & 3.2 & 170 & 88 \\
\hline
\end{tabular}

Table 5

Results of the range analysis.

\begin{tabular}{llll}
\hline Index & $1\left(I_{\mathrm{SL}}\right)$ & $2\left(T_{\mathrm{S}}\right)$ & $3\left(I_{\mathrm{ST}}\right)$ \\
\hline$I_{j} / k_{j}$ & 74.3333 & 75.6667 & 80 \\
$I I_{j} / k_{j}$ & 77 & 78 & 76.6667 \\
$I I I_{j} / k_{j}$ & 82.3333 & 80 & 77 \\
$D_{j}$ & 80 & 4.3333 & 3.3333 \\
\hline
\end{tabular}

experiments could yield the products with satisfactory quality. Therefore, the stable operating point range was so large that the parameters could be easily matched.

As shown in Table 4, the lowest welding quality was obtained from experiment 2 , whereas the highest welding quality was obtained from experiment 9 . The current waveforms and welding seams obtained from these experiments are shown in Figs. 10-13 .

First, the stability of the welding process could be analyzed using current waveform. According to Fig. 10, which showed the current waveform graph of experiment 2 , this experiment showed no distinct arc interruption or short circuit. Additionally, the welding process was unsteady and juts appeared. Moreover, explosion could occur during the process and some expulsions may occur. In Fig. 11 which shows the welding seam, although the starting arc process was smooth, the welding seam was irregular, and undercut and arc interruption could occur. However, due to the existence of a transition period, the process could independently retrieve a normal state. The appearance of the welding seam was regular. Moreover, a transition zone existed in the welding seam, but the zone was interrupted when the tendency or arc interruption appeared.

On the other hand, the current waveform of experiment 9 shown in Fig. 12 was regular and steady, with distinct transition period. The corresponding welding seam shown in Fig. 13 was regular without undercut and showed a distinct tendency for arc interruption. The welding seam was smooth, and the transition zone observed in the welding seam crest was well-formed with silver gloss.

\subsubsection{Range analysis results}

In general case, the range analysis method and the variance method were used to analyze the orthogonal experimental process. The following results could be obtained from the range analysis:

(1) The effects of each parameter on the experimental results.

(2) The variation tendency of the experimental results for each parameter. To directly observe the variation tendency, the calculated results could also be described in figures. 


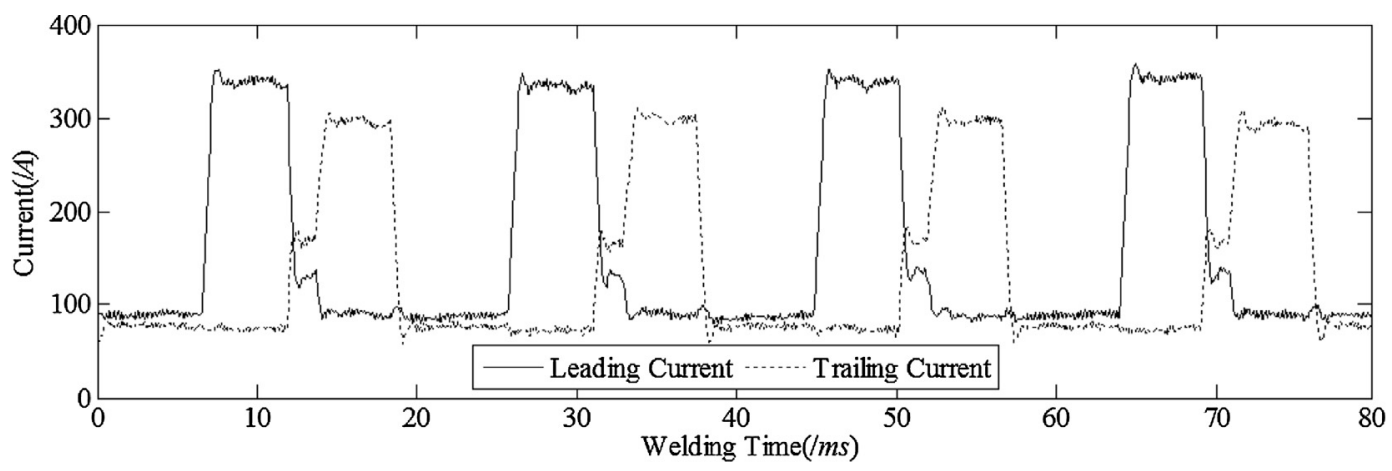

Fig. 10. Current waveform of experiment 2.

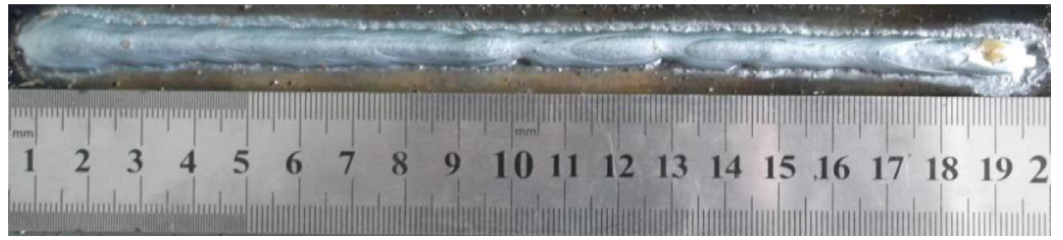

Fig. 11. Welding seam of experiment 2 .

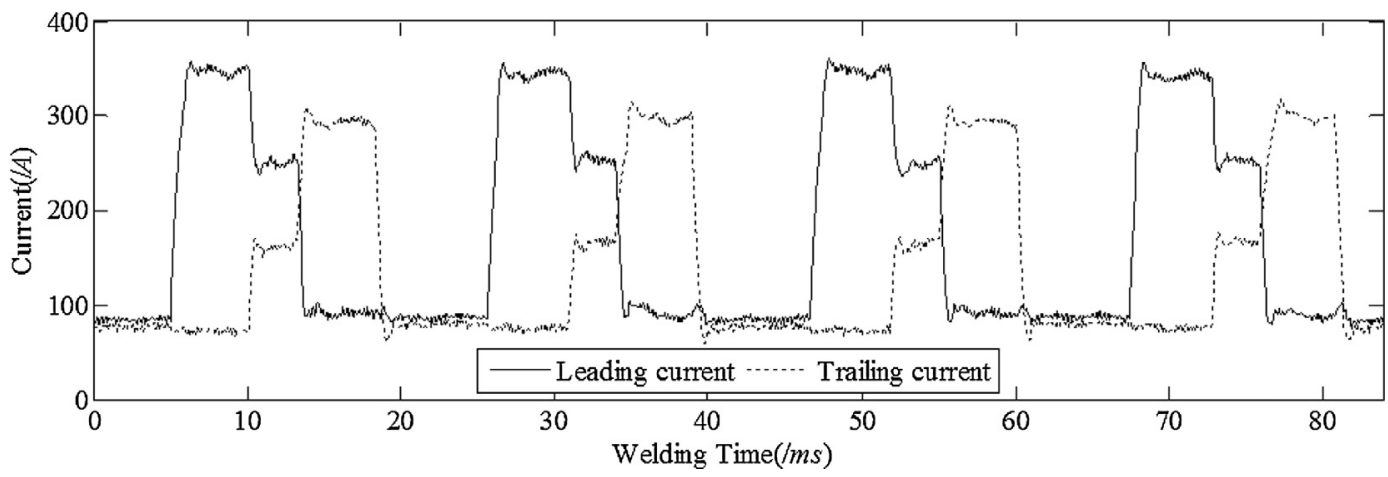

Fig. 12. Current waveform of experiment 9.

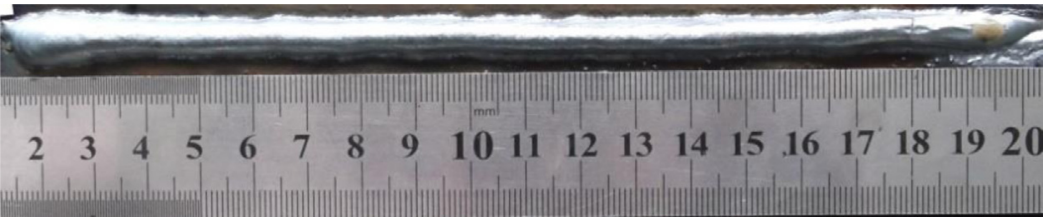

Fig. 13. Welding seam of experiment 9 .

(3) The optimum parameters combination that could achieve the best performance.

In this work, the range analysis method was employed. The analysis results are shown in Table 5 . In this table, the data in $I_{j}$ and $I I_{j}$, $\mathrm{III}_{j}$ represent the sum of the experimental results at level 1 , level 2 and level $3 ; k_{j}$ is the number of replicates of the same level in the $j$ th column, in this work, $k_{j}=3 . I_{j} / k_{j}, I I_{j} / \mathrm{k}_{j}, I I I_{j} / k_{j}$ are the mean values of the experimental results corresponding to each level in the $j$ th column; $\mathrm{D}_{j}$ is the range, which is calculated using the following equation:

$D_{j}=\max \left\{\frac{I_{j}}{k_{j}}, \frac{I I_{j}}{k_{j}}, \ldots \ldots\right\}-\min \left\{\frac{I_{j}}{k_{j}}, \frac{I I_{j}}{k_{j}}, \ldots \ldots\right\}$

According to Table 5, the effect of the leading transition current $I_{\mathrm{SL}}$ on welding quality was the most significant, then followed by that of the transition time $T_{\mathrm{S}}$, and the effect of $I_{\mathrm{ST}}$ was relatively the

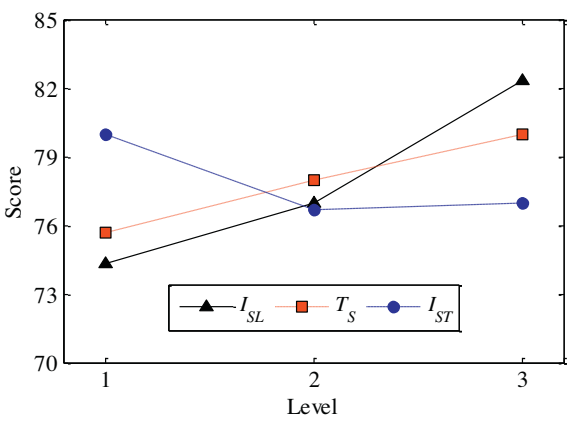

Fig. 14. Parameters effect curves. 


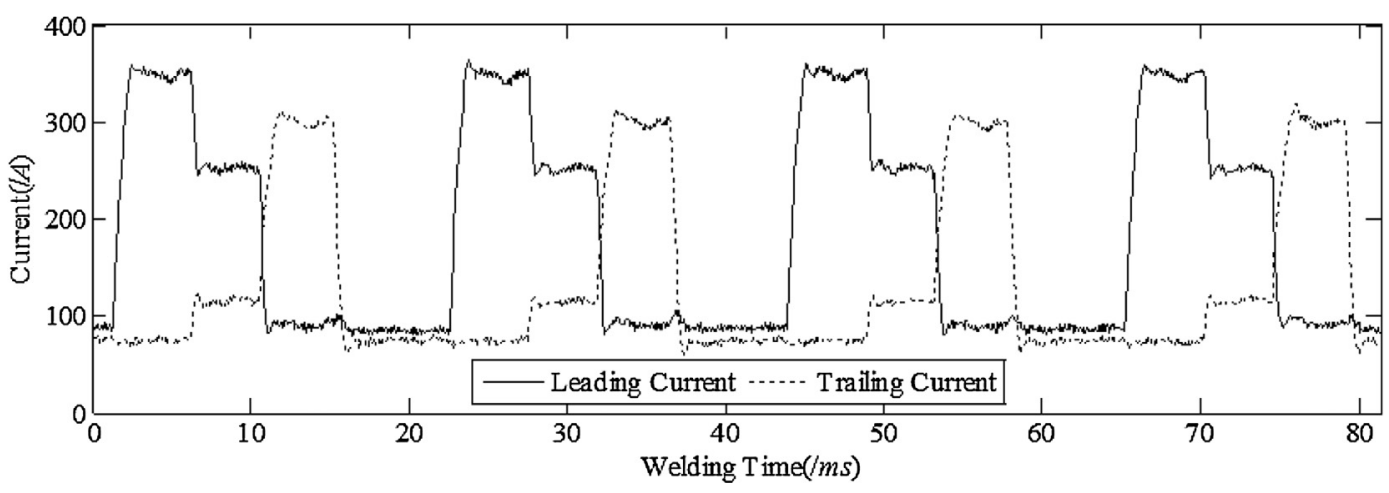

Fig. 15. Current waveform under the optimized parameters.

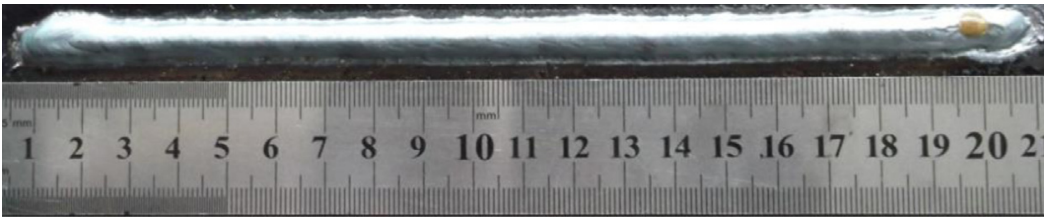

Fig. 16. Weld surface under the optimized parameters.

smallest. Fig. 14 shows the effects of these three parameters on the welding process.

According to Fig. 14, the welding quality was much better as increasing $I_{\mathrm{SL}}$ and $T_{\mathrm{S}}$, while the welding quality was the best when the value of $I_{\mathrm{ST}}$ was smallest, and larger value of the $I_{\mathrm{ST}}$, worse welding quality could be obtained. In addition, when the current in transition period was not very large, relative satisfactory welding quality could be obtained. Because the variation difference of current was lowered by means of existence of transition period, a much steadier welding process could be obtained. Hence, increase the transition period can improve the welding quality in practice.

\subsection{Experiment verification}

According to the criteria of evaluating welding seam quality, in this orthogonal experiment, high experimental index scores indicate high welding quality. Hence, the optimal operating condition is a combination of the parameters that can increase the welding quality scores in each level. As shown in Table 5, the average score of $I_{\mathrm{SL}}$ in level 3 was highest, the corresponding value was 82.333; the highest average scores of $T_{S}$ for each level also appeared in level 3 , the value was 80 ; the highest average scores of $I_{S T}$ for each level corresponded to the level 1 . Then the optimum parameters combination can be obtained using each level which corresponded the highest score for each parameter. According to Tables 3 and 5, the optimum parameters combination can be shown as follows:

Leading transition current $I_{\mathrm{SL}}$ at level 3: $250 \mathrm{~A}$;

Transition time $T_{\mathrm{S}}$ at level 3: $3.2 \mathrm{~ms}$;

Trailing transition current $I_{\mathrm{ST}}$ at level 1: $120 \mathrm{~A}$.

Thus, a corresponding experiment could be conducted to validate the above-described outcome. The current waveform and welding seam are shown in Figs. 15 and 16.

As shown in Fig. 15, the current waveform was highly regular with distinct symmetrical transition period, and the waveform appeared was very steady without any short circuit or arc interruption. The sound heard during the experiment was a gentle sound ranging between "woon" and "zi.". According to Fig. 16, the welding seam shape was also regular, and no undercut, arc interruption, jut or other defects appeared. Additionally, the surface was much cleaner than that observed without a transition period.

\subsection{Discussion}

According to the experimental results, the effects of $I_{\mathrm{SL}}, T_{\mathrm{S}}$ and $I_{\mathrm{ST}}$ on the welding quality presents some certain rules. However, the current researches were based on the individual varying of these elements, instead of concerning the effects when all of three parameters vary at the same time. Due to the complexity of the welding process, the effect when all the parameters vary on the welding quality may have large differences. Hence, the future work will concern this point and explore the effect in depth.

In addition, the workpiece in this work is steel. In the future work, the optimum combination of control variables for current waveform control in the transition period will be further examined under workpieces with other materials and corresponding other currents.

Furthermore, the double-wire MIG process is very complex. To decrease the complexity of parameters matching and improve the intelligent control level of the process, the relations between different parameters will be further explored. The analytical equations will be obtained through further theoretical and experimental analysis, and then an intelligent parameter matching library will be established to serve different applying occasions.

\section{Conclusions}

According to analyze the effect discipline of electromagnetic force on double-wire MIG welding, a symmetrical transition waveform control method was proposed to improve the welding quality, relative experiments were conducted, then some conclusions were drawn as follows:

(1) The method was based on current waveform control. The experimental results showed that it could improve the welding quality, and make the welding process steadier, as well as more polished welding seam could be obtained.

(2) The effects of three important parameters, which were $I_{\mathrm{SL}}, T_{\mathrm{S}}$ and $I_{\mathrm{ST}}$ on the performance were obtained in detail through 
orthogonal experiment and range analysis. It could be concluded that the effect of $I_{\mathrm{SL}}$ on welding quality was the most important significant, then followed by $T_{s}$, whereas the effect of $I_{\mathrm{ST}}$ was relatively small. $T_{\mathrm{S}}$ could improve the stability of welding process within a proper range, and when the $I_{\mathrm{SL}}$ and $I_{\mathrm{ST}}$ had small difference from their original values, the welding quality could be improved.

(3) The optimum parameters combination could be obtained through the results of range analysis for the experiments using different combinations. The experimental results showed that more satisfactory performance can be obtained when the optimum parameters combination was employed.

\section{Acknowledgments}

The authors would like to thank the Foundation for Distinguished Young Teachers' Training of Guangdong (Grant No. Yq2013106), the Characteristics of the Guangdong Province Ordinary University Innovation Project (Grant No. 2014KTSCX145) and Natural Science Foundation of Guangdong Province (2015).

\section{References}

Andersson, J., Erik, T., Joakim, H., 2006. The fundamental stability mechanism in tandem MIG/MAG welding, and how to perform implementation. In: Proceedings of the IIW International Conference, Quebec City, Canada.

Bagus Yudharto Bharotokusumo, Y., Hermans, Marcel Joseph Alarie, Richardson, I.M., 2008. Process stability analysis during tandem wire arc welding. Rev. Soldagem Insp., 13., pp. 82-90, ISSN 0104-9224.

Chandel, R.S., 1987. Mathematical modeling of melting rates for submerged arc welding. Weld. J. 66, 135s-140s.

DeRuntz, B., 2003. Assessing the benefits of surface tension transfer welding to industry. J. Ind. Technol. 19, 55-62.

Feng, J., Zhang, H., He, P., 2009. The CMT short-circuiting metal transfer process and its use in thin aluminium sheets welding. Mat. Des. 30, 1850-1852.

Goecke, S., Berlin, F.U.B.T., Hedegård, J., Joining, S.I.M.R., Ab, E.W.E., 2011. Tandem Mig/Mag Welding, 56 (2-3. A Welding Review Published by Esab, pp. 24-28.

Halmoy, E., 1980. Wire melting rate, droplet temperature and effective anode melting potential. Arc Phys. Weld. Pool Behav., 49-57.

Kawagoe, T., Suzuki, K., 2014. Method and apparatus for arc welding by controlling welding current, U.S.

Keppel, G., 1991. Design and Analysis: A researcher's Handbook. Prentice-Hall, Inc.

Li, K., Zhang, Y., 2010. Interval model control of consumable double-electrode gas metal arc welding process. IEEE Trans. Autom. Sci. Eng. 7, 826-839.

Motta, M.F., Dutra, I.C., Gohr Jr., R., Scotti, A., 2007. A study on out-of-phase current pulses of the double wire MIG/MAG process with insulated potentials on coating applications-part I. J. Braz. Soc. Mech. Sci. Eng. 29, 202-206.

Niu, Y., Xue, H., Li, H., Zeng, Z., 2010. Effect of peak pulse voltage on metal transfer and formation of weld in double-wire pulsed MIG welding (in Chinese). Trans. China Weld. Inst. 31, 50-54.

Kah, P., Suoranta, R., Martikainen, I., 2013. Advanced gas metal arc welding processes. Int. J. Adv. Manuf. Technol. 67, 655-674.
Peltola, T., Kumpulainen, J., Veikkolainen, M., 2010. Novel tailored welding arc help welders meet quality and productivity demands. In: Innovation and Business Development. Kemppi Oy, Lahti, pp. 15-18.

Person, J.A., Ruzek, J.M., 1956. High speed tandem arc working, US patent: 2756311.

Peterson, N., 2009. New technology doubles contractor's pipe welding output. Weld. J. 88, 56-58.

Chandel, R.S., 1988. Wire melting rate in mild steel MIG welding. Metal Constr. 20 214-216.

Petro, S.J., 2011. Effect of Interpass Temperature on the Structure and Properties of Multipass Weldments in High Performance Nickel Alloys. Colorado State University.

S.R. Peters, 2013. Arc welding with waveform control function, U.S. patent: US20140251967A1.

Reis, R.P., Souza, D., Ferreira Filho, D., 2015. Arc interruptions in tandem pulsed gas metal arc welding. J. Manuf. Sci. Eng. 137 (1), 011004.

Schierl, A., 2005. The CMT process a revolution in welding technology. International Conference, Benefits of New Methods and Trends in Welding to Economy, Productivity and Quality.

Shi, C., Zou, Y., Zou, Z., Wu, D., 2014a. Twin-wire indirect arc welding by modeling and experiment. J. Mater. Process. Technol. 214, 2292-2299.

Shi, C., Zou, Y., Zou, Z., Zhang, H., 2014b. Physical characteristics of twin-wire indirect arc plasma. Vacuum 107, 41-50.

Steinert, E.F., 1954. Twin arc welding system, US patent: 2673915.30.

Suban, M., Tušek, I., 2001. Dependence of melting rate in MIG/MAG welding on the type of shielding gas used. J. Mater. Process. Technol. 119, 185-192.

Ashton, T., 1954. Twin-arc submerged arc welding. Weld. J. 33, 350-355.

Taguchi, G., Clausing, D., Watanabe, L.T., 1987. System of Experimental Design: Engineering Methods to Optimize Quality and Minimize Costs. UNIPUB/Kraus International Publications, White Plains, NY

Tong, H., Ueyama, T., 2011. Feature of low frequency modulated type pulsed MIC welding process. Weld. Join. 11, 33-35.

Tušek, J., 2000. Mathematical modeling of melting rate in twin-wire welding. J. Mater. Process. Technol. 100, 250-256.

Ueyama, T., Ohnama, T., Yamazaki, K., Tanaka, M., Ushio, M., Nakata, K., 2005. High-speed welding of steel sheets by the tandem pulsed gas metal arc welding system. Trans. JWRI 34, 11-18.

Ueyama, T., Ohnawa, T., Tanaka, M., Nakata, K., 2007. Occurrence of arc interaction in tandem pulsed gas metal arc welding. Sci. Technol. Weld. Join. 12, 523-529.

Ueyama, T., Uezono, T., Era, T., Tanaka, M., Nakata, K., 2009. Solution to problems of arc interruption and arc length control in tandem pulsed gas metal arc welding. Sci. Technol. Weld. Join. 14, 305-314.

Uusitalo, J., 2007. Modified short arc process- new way of welding root passes. Weld. World (Lond.) 51, 283.

Wang, F., Hua, X.M., Ma, X.L., Wu, Y., Cao, N., Qian, W., 2011. Analysis of arc interference and interruption in double-wire GMAW welding. Trans. China Weld. Inst. 32 (7), 109-112.

Wen, Y., Huang, S., Wu, K., Miu, Z., 2010. Effects of current phase relations on weld bead formation for twin-wire co-pool pulsed MAG welding (in Chinese). Trans. China Weld. Inst. 31, 17-20.

Yao, P., Xue, J., Meng, W., Zhu, S., 2009. Influence of processing parameters on weld forming in double pulse MIG welding of aluminum alloy (in Chinese). Trans. China Weld. Inst. 30, 69-72

Yao, P., Xue, J., Ma, Q., Chen, H., Chen, X., 2012. Symmetrical transition waveform control process of double wire MIG welding (in Chinese). Trans. China Weld. Inst. 33, 21-24.

Yao, P., Xue, J., Wang, L., Zhu, Q., 2014a. Fuzzy evaluation of weld quality based on Matlab. China Weld. 23, 67-71.

Yao, P., Xue, I., Zhou, K., Wang, X., 2014b. Sample entropy-based approach to evaluate the stability of double-wire pulsed MIG welding. Math. Prob. Eng. 2014,1 . 